\title{
Phase-change Optical Nonlinearity as a Cellular Automaton
}

\author{
L. Zhang ${ }^{1,2}$, K. F. MacDonald ${ }^{1}$ and N. I. Zheludev ${ }^{1,3}$ \\ 1. Optoelectronics Research Centre and Centre for Photonic Metamaterials, University of Southampton, \\ Highfield, Southampton, SO17 1BJ, UK \\ 2. School of Mathematics and Physics, Anqing Normal University, Anqing 246133, P. R. China \\ 3. Centre for Disruptive Photonic Technologies, School of Physical and Mathematical Sciences and The Photonics Institute, \\ Nanyang Technological University Singapore, 637378, Singapore
}

We introduce a cellular automata methodology for studying the photonics of light-induced phase transitions. A model governed by a sparse set of evolutionary rules successfully describes the complex, non-stationary, spatially inhomogeneous dynamics and nonlinear optical properties of a medium undergoing a light-induced structural transition.

Light-induced phase changes are some of the most important processes in photonics. They are exploited in optical data storage and laser-based manufacturing, and are now intensely explored for a range of applications in active, reconfigurable nanophotonic devices. However, comprehensive modelling of photonic phase change functionality is extremely challenging as it involves entangled processes of atomic/molecular structural change, domain and crystallization dynamics, change of optical properties in inhomogeneous media, and the transport/dissipation of heat and light, evolving on disparate time and length scales. We show that the problem of describing such multiphysical complexity can be reduced to one of just a few free parameters and transition rules in a classic cellular automata model.

We consider the example of a polymorphic gallium near to its bulk melting transition - a system in which optical excitation may proceed via both thermal and non-thermal mechanisms, and in which surfaces are subject to nanoscale 'pre-melting'. The cellular model reproduces experimentally observed transition and relaxation dynamics for excitation pulse durations ranging from $f s$ to $\mu$ s and provides insight to the microscopic mechanisms of transition without recourse to deep understanding or analytical description of atomic dynamics, band structure, electromagnetics, thermodynamics or nanoscale energy conservation.

The cellular automata approach may be applied to a variety phase-change, nonlinear optical and active (nano)photonic systems. It has considerable heuristic value for the study of complex nonlinear optical processes and non-equilibrium behaviours in systems close to a transition point, which cannot be described analytically. 